\title{
Antiphospholipid syndrome and pregnancy
}

\section{Opinion}

Research regarding the pathogenesis of vascular damage and blood coagulation disorders is a constant challenge in modern medicine. With the introduction of immunological research in the wide clinical practice, a significant expansion of the knowledge about the role of immunopathological processes in various diseases has been achieved. The development of various forms of organic pathology in these patients is linked to the overproduction of a wide range of organ-specific antibodies that react with antigens in the nucleus, cytoplasm, cell membrane, and serum proteins. This process ends with the formation of a large number of antibodies. In recent decades, a number of publications have been published on antiphospholipid antibodies (aPls) and their role in the formation of the clinical entity in patients with autoimmune diseases. aPLs are negatively charged antibodies against phospholipids and their occurrence is associated with a heterogeneous clinical manifestation known as Antiphospholipid syndrome. In hematology, this syndrome is associated with thrombosis and thrombocytopenia, in neurology, it is associated with ischemic brain disease, in cardiology - with vascular disease, in dermatology - with Livedo reticularis, and in obstetrics it is associated with unsuccessful pregnancy, e.g. fetal growth restriction and fetal loss (recurrent miscarriage). ${ }^{1,2}$

Antiphospholipid syndrome (APS) is defined as an autoimmune disease with the presence of aPLs (positive anticardiolipn and/ or lupus anticogulans test) associated with clinical manifestations of arteriovenous thrombosis, recurrent miscarriages, and thrombocytopenia. ${ }^{2,3}$

Antiphospholipid antibodies (APL) are: ${ }^{4}$

a. Anticardiolipin antibodies (aCL)

b. Lupus Anticoagulants (LA)

c. anti- $\beta_{2}$ glycoprotein I antibodies (anti- $\beta_{2}$ GPI).

Regarding the pathogenesis, APS still doesn't have any known etiology. However, there are several hypotheses to explain the probable cause, such as:

i. Passive transfer of maternal antibodies in the fetus and newborn.

ii. Familial occurrence of aPL lead to genetic association which includes DR7, C4 null allele, DRw53 and HLA-DR4.

iii. Infective etiology: phospholipid molecules are ubiquitous in nature and are present in the inner surface of the cell and in microorganisms. Therefore, during infectious disease processes, including viral, bacterial and parasitic agents, the disruption of cellular membranes may occur during cell damage. PLs release and stimulate aPL antibodies. ${ }^{5}$

The reasons behind vascular thrombosis are still unclear. The "two hit" hypothesis suggests that the "first hit" may be oxidative stress, surgery, trauma, or infections leading to endothelia cell damage. This exerts different effects on endothelial cells, monocytes, platelets, and complement. Binding and activation of these cell types causes an increased expression of adhesion molecules, secretion of cytokines, and production of arachidonic acid metabolites. aPL may
Volume 12 Issue 5 - 202I

\author{
Jasovic-Siveska Emilija,' Simeonova-Krstevska \\ Slagjana, ${ }^{2}$ Milkovski Daniel ${ }^{2}$ \\ 'Obstetric and Gynecology, PHO Medihelp, University St. \\ Clement of Ohrid, Bitola, North Macedonia \\ ${ }^{2}$ University Clinic of Obstetrics and Gynecology, Medical Faculty, \\ Skopje, North Macedonia
}

Correspondence: Jasovic-Siveska Emilija, Obstetric and Gynecology, PHO Medihelp, University St. Clement of Ohrid, Bitola, North Macedonia, Tel +38970782100 , Email medihelp@t-home.mk

Received:September 29, 2021 | Published: October 15, 2021

also participate in oxidant-mediated injury to vascular endothelium or bind to perturbed cells that lose their regular membrane symmetry and express anionic phospholipids on their surface. The interaction of antibodies with clotting regulation such as prothrombin, factor $\mathrm{X}$, protein $\mathrm{C}$, and plasmin might hinder inactivation of procoagulant factors and impede fibrinolysis. In pregnancy, placental thrombosis and fetal loss may result from interference with annexin A5, a natural anticoagulant. Abnormalities in placentation leading to pregnancy loss may result from antibodies binding leading to a reduction of human chorionic gonadotropin secretion or triggering an inflammatory response resulting in trophoblast damage. ${ }^{6}$

The most common finding in patients with APS is placental thrombosis, although it is not present in all and may not always explain intrauterine fetal death.

The pathophysiological mechanism in women with miscarriages or intrauterine fetal death is not always the only disorder. Phospholipid molecules are an integral part of every cell membrane, and their role is in the cellular transmission of signals, which in turn participates in the division of cells and their secretory function. There is indirect evidence that aPLs inhibit phospholipase A2 and phospholipase C. These phospholipases stimulate the production of glycoprotein hormones on the placenta HCG and HPL. IgG antibodies cross the placental barrier and thus can reach the fetus, but so far no fetal thrombosis and death have been reported as a result of said occurrence. For now, it can be concluded that in children born to pregnancies where the mother suffered from APS, complications related to prematurity may occur. $^{7}$

In gynecology and obstetrics there is a growing interest in APS and APL because these antibodies are often associated with early miscarriages, missed abortions and/or fetal death in the second and third trimesters of pregnancy, but also the occurrence of chorea gravidarum, preeclampsia, ovarian hypofunction, hyperandrogenism, 
endometriosis, and there are reports that APL is also found in the follicular fluid in patients undergoing in vitro fertilization. ${ }^{8-10}$

Regarding the definition, G.R.V. Hughes et al., introduced clinical and laboratory criteria in the diagnosis of APS: ${ }^{4,7}$

\section{Clinical criteria:}

Basic: thrombosis, obstetric pathology, thrombocytopenia.

Additional: livedo reticularis, nervous system disorders, ischemic bone necrosis, chronic lower leg ulcer, endocarditis.

\section{Serological criteria:}

a. ACL class IgG and / or class IgM (isotype)

b. LA

c. Anti- $\beta_{2}$ GPI antibody of IgG and / or IgM isotype.

Indications for determining APLs are: infertility, clinical suspicion or active systemic lupus erythematosus, deep vein or arterial thrombosis, positive antinuclear antibody test, platelet count below $175 \times 10^{9}$ false positive VDRL test, positive test antibody test against smooth muscles. ${ }^{4,7}$

After the association between APS and unsuccessful pregnancies was noted, therapy was started for these patients. There are different approaches to the treatment of APS and often the therapy is adapted to the patient, so it could be said that treatment is more of an art than the application of an established protocol with specific doses of the drugs. The most common use is the combined use of drugs.

The first reports of APS treatment indicated the usage of corticosteroids, namely prednisone in high doses. Although in most cases, all reports were positive, they referred to a small number of patients. Shortly afterwards, there were reports of side effects from such therapy (Cushing-like appearance of the patient, acne, diabetes, hypertension, osteoporosis, etc.). ${ }^{11}$

Low-dose aspirin has been shown to be a very useful drug in preventing intrauterine fetal growth restriction and preeclampsia. Patients under low-dose aspirin therapy showed no side effects. ${ }^{4}$

Daily subcutaneous administration of heparin from prophylactic to therapeutic doses can prevent placental thrombosis and consequential fetal loss. Low molecular weight heparin is widely used today for a wide range of benefits. ${ }^{11}$

In case of proven autoimmune disease, administration of preconceptional immunotherapy can be used as immunosuppressive therapy. The use of plasmapheresis and azathioprine has been reported in the literature as an adjunct to basic therapy. Cases have been described in which no therapeutic method was used, i.e. the patients were not treated, and still had a successful pregnancy outcome. ${ }^{11,12}$

The normal pregnancy and the birth of a healthy child transcends the medical aspects as it delves deeper into the most sensitive layers: the social, demographic and other aspects of each nation and state. The large number of artificial abortions, whose exact number is unknown, as well as the high percentage of couples who face the problem of having a healthy child, require the discovery of every possible cause that hinders the pregnancy and its normal course. Particular attention should be paid to patients with recurrent miscarriage or intrauterine fetal death. In patients with such medical history, despite excluding the numerous possible causes (infections, endocrinological factors, anatomical abnormalities, genetic disorders, etc.), a possible immune disorder or autoimmune disease should be considered.

APS today is a field of interest of the entire clinical medicine given the richness of clinical manifestations. This multidisciplinary in the diagnostic therapeutic approach is at the same time a reflection of the indivisibility of medicine in the approach to the patient and with one goal: to treat the patient, not the disease.

\section{Acknowledgments}

None.

\section{Funding}

None.

\section{Conflicts of interest}

The authors did not report any potential conflicts of interest.

\section{References}

1. Giannakopoulos B, Krilis SA. How I treat the antiphospholipid syndrome Blood. 2009;114(10):2020-2030.

2. Garcia D, Erkan D. Diagnosis and management of the antiphospholipid syndrome. $N$ Engl J Med Overseas Ed. 2018;378:2010-2021.

3. Ching Soh M, Nelson-Piercy C. Antiphospholipid syndrome in pregnancy. Expert Review of Obstetrics \& Gynecology. 2010;5:6:741-761.

4. Antovic A, Sennström M, Bremme K, et al. Obstetric antiphospholipid syndrome. Lupus Science \& Medicine. 2018;5:e00197.

5. Di Prima FA, Valenti O, Hyseni E, et al. Antiphospholipid syndrome during pregnancy: the state of the art. J Prenat Med. 2011;5(2):41-53.

6. Khangura Raminder Kaur, Cooper Shontreal, Luo Guo-Yang. Antiphospholipid antibody syndrome: pathogenesis, diagnosis, and management in pregnancy. Maternal-Fetal Medicine. 2019;1(1):38-42.

7. Abrahams VM, Chamley LW, Salmon JE. Emerging treatment models in rheumatology: antiphospholipid syndrome and pregnancy: pathogenesis to translation. Arthritis Rheumatol. 2017;69(9):1710-1721.

8. Boddi M, Prisco D, Fredi S, et al. Antiphopholipid antibodies and pregnancy disorders in women with insulin dependent diabetes. Thromb Res. 1996;82(3):207-216.

9. Uncu G, Ozan H, Kucukerdogan J, et al. Anticardiolipin antibodies in pregnancy induced hypertension. Eur J Obstet Gynecol Reprod Biol. 1996;70(1):97-100

10. Zhang S, Dong J, Liu B. Evaluation and its clinical significance of antiplatlet granule membrane protein-140 autoantibodies and anti-calmudolin antibody in patients with sever pregnancy induced hypertension. Chung Hua Fu Chan Ko Tsa Chih. 1995;30(11):665-667.

11. Ghembaza A, Saadoun D. Management of antiphospholipid syndrome. Biomedicines. 2020;8(11):508.

12. Lubbe WF, Butler WS, Palmer SJ, et al. Fetal survival after prednisone suppression of maternal lupus-anticoagulant. Lancet. 1983;i:1361-1363. 